\title{
Deuteron electromagnetic form factors in a renormalizable formulation of chiral effective field theory
}

\author{
E. Epelbaum ${ }^{1}$, A.M. Gasparyan ${ }^{1,2}$, J. Gegelia ${ }^{1,3, a}$, and M.R. Schindler ${ }^{4}$ \\ 1 Institut für Theoretische Physik II, Fakultät für Physik und Astronomie, Ruhr-Universität Bochum 44780 Bochum, Germany \\ 2 SSC RF ITEP, Bolshaya Cheremushkinskaya 25, 117218 Moscow, Russia \\ 3 Tbilisi State University, 0186 Tbilisi, Georgia \\ 4 Department of Physics and Astronomy, University of South Carolina, Columbia, SC 29208, USA
}

Received: 7 January 2014 / Revised: 4 February 2014

Published online: 3 March 2014

(C) The Author(s) 2014. This article is published with open access at Springerlink.com

Communicated by J. Bijnens

\begin{abstract}
We calculate the deuteron electromagnetic form factors in a modified version of Weinberg's chiral effective field theory approach to the two-nucleon system. We derive renormalizable integral equations for the deuteron without partial wave decomposition. Deuteron form factors are extracted by applying the Lehmann-Symanzik-Zimmermann reduction formalism to the three-point correlation function of deuteron interpolating fields and the electromagnetic current operator. Numerical results of a leading-order calculation with removed cutoff regularization agree well with experimental data.
\end{abstract}

\section{Introduction}

The seminal papers by Weinberg on chiral effective field theory (EFT) of nuclear forces [1,2] have triggered an intense activity starting with ref. [3]. For recent reviews see, e.g., refs. [4,5]. One of the most discussed aspects of the application of chiral effective field theory to twoand few-body problems is related to the question of how to properly renormalize the resulting integral equations. A new framework to solve this problem was proposed in ref. [6], which is based on the manifestly Lorentzinvariant effective Lagrangian and time-ordered perturbation theory. Within this scheme the leading-order (LO) nucleon-nucleon scattering amplitude is obtained by solving an integral equation (known as the Kadyshevsky equation [7]), and corrections are calculated perturbatively. The LO equation is perturbatively renormalizable due to the milder ultraviolet behavior of the two-nucleon propagator compared to the standard heavy-baryon formalism. Partial wave projected equations have unique solutions except for the ${ }^{3} P_{0}$ wave, which requires a special treatment. In the present study we calculate the electromagnetic form factors of the deuteron, and therefore the issue of the ${ }^{3} P_{0}$ wave is not relevant here.

The electromagnetic structure of the deuteron has been extensively analyzed in the EFT framework using various approaches. In particular, Kaplan, Savage and Wise [8] calculated the electromagnetic form factors of the

\footnotetext{
a e-mail: gegelia@kph.uni-mainz.de
}

deuteron up to next-to-leading order (NLO) in a framework based on a perturbative treatment of potential pions and found good agreement with data up to momentum transfers of the order of $q \sim 400 \mathrm{MeV}$. Shortly thereafter a number of calculations based on Weinberg's approach (or variations thereof) with non-perturbative pions have been performed at various orders in the chiral expansion [9-13], see also refs. [14,15] for pioneering quantitative studies of nucleon-nucleon scattering in this framework and refs. $[4,5]$ for recent review articles. Generally, after employing factorization in order to account for single-nucleon electromagnetic structure, a good description of the deuteron form factors up to rather high values of the momentum transfer was reported in all these calculations provided the isoscalar single-nucleon form factors are accurately described ${ }^{1}$. Two-body currents, worked out at leading loop order in the heavy-baryon formulation of chiral EFT by Park et al. [16] and rederived recently by the JLab-Pisa [17-19] and Bochum-Bonn groups [20,21], are mainly of isovector type and thus play only a minor role for the deuteron. Further applications of the exchange currents to the electromagnetic structure and reactions in two- and three-nucleon systems are reported in refs. [22, 23]. With the exception of ref. [8], which makes use of dimensional regularization, all these calculations employ a finite ultraviolet cutoff $\Lambda$ chosen to be smaller or of the

\footnotetext{
1 Note that the factorization amounts to taking into account higher-order terms in the chiral expansion of the single-nucleon current operator.
} 
order of the rho-meson mass. Much larger cutoff values in the range of $\Lambda \lesssim 4 \mathrm{GeV}$ are considered in ref. [24] together with the leading- and next-to-next-to-leading-order chiral wave functions.

In the present work, we extend our recently suggested renormalizable formulation of nuclear chiral EFT with non-perturbative pions [6] to calculate the electromagnetic form factors of the deuteron at lowest order. Similarly to ref. [25], we derive a system of integral equations for the deuteron without making use of partial wave decomposition. The crucial new feature of our framework is its explicit renormalizability in spite of the nonperturbative treatment of the one-pion-exchange (OPE) potential. This allows us to take the cutoff parameter to infinity when calculating the two-nucleon amplitude both perturbatively as well as non-perturbatively. Our paper is organized as follows. In sect. 2 we briefly outline a general formalism to calculate the form factors of the deuteron in quantum field theory. The integral equations for the deuteron interpolating field interacting with a pair of nucleons are worked out in sect. 3. Finally, a discussion and summary of the obtained results are given in sect. 4 .

\section{The deuteron form factors}

We use the conventions and notations of ref. [8]. The deuteron is characterized by its momentum $P^{\mu}$ and polarization $\epsilon^{\mu}$. The polarization vectors can be expanded in terms of basis vectors $\epsilon_{i}^{\mu}(i=1,2,3)$, which satisfy the conditions

$$
P_{\mu} \epsilon_{i}^{\mu}=0, \quad \epsilon_{i \mu}^{*} \epsilon_{j}^{\mu}=-\delta_{i j}, \quad \sum_{i=1}^{3} \epsilon_{i}^{* \mu} \epsilon_{i}^{\nu}=\frac{P^{\mu} P^{\nu}}{M_{d}^{2}}-g^{\mu \nu},
$$

where $M_{d}=2 m-B$ is the deuteron mass, $B$ its binding energy, and $m$ the nucleon mass. We choose these polarization vectors so that in the rest frame of the deuteron $\epsilon_{i}^{\mu}=\delta_{i}^{\mu}$ and denote deuteron states with $|\mathbf{P}, i\rangle\left(\equiv\left|\mathbf{P}, \epsilon_{i}^{\mu}\right\rangle\right)$. These states satisfy the normalization condition

$$
\left\langle\mathbf{P}^{\prime}, j \mid \mathbf{P}, i\right\rangle=\frac{P^{0}}{M_{d}}(2 \pi)^{3} \delta^{3}\left(\mathbf{P}-\mathbf{P}^{\prime}\right) \delta_{i j} .
$$

The matrix element of the electromagnetic current operator to leading order in a non-relativistic expansion can be parameterized as

$$
\begin{aligned}
\left\langle\mathbf{P}^{\prime}, j\left|J_{e m}^{0}\right| \mathbf{P}, i\right\rangle= & e\left[F_{C}\left(q^{2}\right) \delta_{i j}+\frac{1}{2 M_{d}^{2}} F_{Q}\left(q^{2}\right)\right. \\
& \left.\times\left(\mathbf{q}_{i} \mathbf{q}_{j}-\frac{1}{3} q^{2} \delta_{i j}\right)\right] \\
\left\langle\mathbf{P}^{\prime}, j\left|J_{e m}^{k}\right| \mathbf{P}, i\right\rangle= & \frac{e}{2 M_{d}}\left[F_{C}\left(q^{2}\right) \delta_{i j}\left(\mathbf{P}+\mathbf{P}^{\prime}\right)^{k}+F_{M}\left(q^{2}\right)\right. \\
& \times\left(\delta_{j}^{k} \mathbf{q}_{i}-\delta_{i}^{k} \mathbf{q}_{j}\right)+\frac{1}{2 M_{d}^{2}} F_{Q}\left(q^{2}\right) \\
& \left.\times\left(\mathbf{q}_{i} \mathbf{q}_{j}-\frac{1}{3} q^{2} \delta_{i j}\right)\left(\mathbf{P}+\mathbf{P}^{\prime}\right)^{k}\right]
\end{aligned}
$$

where $\mathbf{q}=\mathbf{P}^{\prime}-\mathbf{P}$ is the transferred momentum and $q=$ $|\mathbf{q}|$. The form factors are normalized as follows (see, e.g., ref. [26]):

$$
F_{C}(0)=1, \quad \frac{e}{2 M_{d}} F_{M}(0)=\mu_{M}, \quad \frac{1}{M_{d}^{2}} F_{Q}(0)=\mu_{Q},
$$

with $\mu_{M}=0.8574(e /(2 m))$ being the deuteron magnetic moment [27] and $\mu_{Q}=0.2859 \mathrm{fm}^{2}$ its quadrupole moment $[28,29]$. It is also common to parameterize the matrix elements of the current operators in terms of the three form factors $G_{C}, G_{M}$, and $G_{Q}$, where

$$
\begin{aligned}
G_{C}\left(q^{2}\right) & =F_{C}\left(q^{2}\right), \\
G_{M}\left(q^{2}\right) & =F_{M}\left(q^{2}\right), \\
G_{Q}\left(q^{2}\right) & =\frac{1}{M_{d}^{2}} F_{Q}\left(q^{2}\right) .
\end{aligned}
$$

We follow ref. [8] and define the deuteron interpolating field as

$$
\begin{aligned}
& \mathcal{D}_{i} \equiv N^{T} \mathcal{P}_{i} N=\sum_{\alpha, \beta, a, b=1}^{2} N_{\alpha, a} \mathcal{P}_{i, a, b}^{\alpha \beta} N_{\beta, b}, \\
& \mathcal{P}_{i} \equiv \frac{1}{\sqrt{8}} \sigma_{2} \sigma_{i} \tau_{2},
\end{aligned}
$$

where $\alpha, \beta$ and $a, b$ are spin and isospin indices, respectively. This choice is made for convenience, and observables do not depend on the particular form of the interpolating field. The full propagator $G_{\mathcal{D}}$ is given by the timeordered product of two interpolating fields,

$$
\begin{aligned}
G_{\mathcal{D}}(P) \delta_{i j} & =\int \mathrm{d}^{4} x e^{-i P x}\left\langle 0\left|T\left[\mathcal{D}_{j}^{\dagger}(x) \mathcal{D}_{i}(0)\right]\right| 0\right\rangle \\
& =\delta_{i j} \frac{i \mathcal{Z}\left(P^{2}\right)}{P^{2}-M_{d}^{2}+i \epsilon} .
\end{aligned}
$$

The electromagnetic form factors of the deuteron are related to the three-point function of the electromagnetic current operator $J_{e m}^{\mu}$ and two interpolating fields,

$$
\begin{aligned}
& G_{i j}^{\mu}\left(P, P^{\prime}\right)= \\
& \int \mathrm{d}^{4} x \mathrm{~d}^{4} y e^{-i P y} e^{i P^{\prime} x}\left\langle 0\left|T\left[\mathcal{D}_{j}^{\dagger}(x) J_{e m}^{\mu}(0) \mathcal{D}_{i}(y)\right]\right| 0\right\rangle,
\end{aligned}
$$

through the Lehmann-Symanzik-Zimmermann (LSZ) reduction formula [8],

$$
\begin{aligned}
& \left\langle\mathbf{p}^{\prime}, j\left|J_{e m}^{\mu}\right| \mathbf{p}, i\right\rangle \\
& =Z\left[G^{-1}(P) G^{-1}\left(P^{\prime}\right) G_{i j}^{\mu}\left(P, P^{\prime}\right)\right]_{P^{2}, P^{\prime 2} \rightarrow M_{D}^{2}} \\
& =-\frac{1}{Z}\left[\left(P^{2}-M_{d}^{2}\right)\left(P^{\prime 2}-M_{d}^{2}\right) G_{i j}^{\mu}\left(P, P^{\prime}\right)\right]_{P^{2}, P^{\prime 2} \rightarrow M_{d}^{2}},
\end{aligned}
$$

where $Z=\mathcal{Z}\left(M_{d}^{2}\right) /\left(2 M_{d}\right)$ is the residue of the propagator. In other words, the form factors can be extracted from the residue of the double pole of the vertex function $G_{i j}^{\mu}\left(P, P^{\prime}\right)$. 

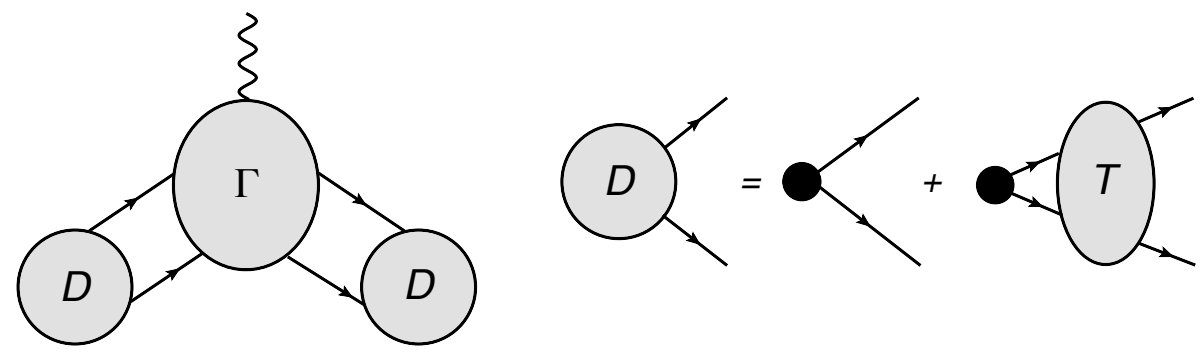

Fig. 1. Vertex function of the electromagnetic current and two interpolating fields of the deuteron. The circle with $\Gamma$ stands for the two-nucleon irreducible part of the vertex function, $D$ is the amplitude of the deuteron interpolating field interacting with a pair of nucleon fields. The black circles represent the interaction vertices of the interpolating field with a pair of nucleons, solid lines represent the nucleons and the waved line corresponds to the electromagnetic current.

The vertex function can be represented diagrammatically as shown in fig. 1. It consists of the two-nucleon irreducible part $\Gamma$ connected to two nucleon-nucleon scattering amplitudes contracted to vertices corresponding to interpolating operators, denoted by $D$. For our LO calculations, we take the irreducible term in the form of the one-nucleon current,

$$
\begin{aligned}
\Gamma^{\mu}\left(p^{\prime}, p\right)= & \frac{F_{1}\left(q^{2}\right)}{2 m}\left(p+p^{\prime}\right)^{\mu} \\
& +i \frac{F_{1}\left(q^{2}\right)+F_{2}\left(q^{2}\right)}{2 m} \delta_{i}^{\mu} \epsilon^{i j k} q^{j} \sigma^{k}
\end{aligned}
$$

where $F_{1}$ and $F_{2}$ are the electromagnetic form factors of the nucleon, $q_{\mu}=p_{\mu}^{\prime}-p_{\mu}$, with $p_{\mu}$ and $p_{\mu}^{\prime}$ the four momenta of incoming and outgoing nucleons, respectively.

\section{The deuteron equation}

In the modified EFT approach of ref. [6] the LO NN scattering amplitude is obtained by solving the integral equation

$$
T\left(\mathbf{p}^{\prime}, \mathbf{p}\right)=V\left(\mathbf{p}^{\prime}, \mathbf{p}\right)-m^{2} \int \frac{\mathrm{d}^{3} \mathbf{k}}{(2 \pi)^{3}} \frac{V\left(\mathbf{p}^{\prime}, \mathbf{k}\right) T(\mathbf{k}, \mathbf{p})}{\omega_{k}^{2}\left(E-2 \omega_{k}+i \epsilon\right)},
$$

where $E=2 \sqrt{\mathbf{p}^{2}+m^{2}}$ denotes the energy of two incoming nucleons in the center-of-mass frame and $\omega_{k}=$ $\sqrt{\mathbf{k}^{2}+m^{2}}$. The LO NN potential can be taken in the usual form

$$
\begin{aligned}
V_{0}\left(\mathbf{p}^{\prime}, \mathbf{p}\right)= & C_{S}+C_{T} \sigma_{\mathbf{1}} \cdot \sigma_{\mathbf{2}} \\
& -\frac{g_{A}^{2}}{4 F^{2}} \tau_{\mathbf{1}} \cdot \tau_{\mathbf{2}} \frac{\sigma_{\mathbf{1}} \cdot\left(\mathbf{p}^{\prime}-\mathbf{p}\right) \sigma_{\mathbf{2}} \cdot\left(\mathbf{p}^{\prime}-\mathbf{p}\right)}{\left(\mathbf{p}^{\prime}-\mathbf{p}\right)^{2}+M_{\pi}^{2}}
\end{aligned}
$$

By parameterizing the potential and the scattering amplitude as (here we indicate explicitly the spin indices omitted in eq. (11))

$$
\begin{aligned}
V_{\alpha \beta, \gamma \delta}\left(\mathbf{p}^{\prime}, \mathbf{p}\right)= & v^{0}\left(\mathbf{p}^{\prime}, \mathbf{p}\right) \delta_{\alpha \gamma} \delta_{\beta \delta} \\
& +v_{a}^{1}\left(\mathbf{p}^{\prime}, \mathbf{p}\right)\left(\sigma_{\alpha \gamma}^{a} \delta_{\beta \delta}+\delta_{\alpha \gamma} \sigma_{\beta \delta}^{a}\right) \\
& +v_{a b}^{2}\left(\mathbf{p}^{\prime}, \mathbf{p}\right) \sigma_{\alpha \gamma}^{a} \sigma_{\beta \delta}^{b},
\end{aligned}
$$

$$
\begin{aligned}
T_{\alpha \beta, \gamma \delta}\left(\mathbf{p}^{\prime}, \mathbf{p}\right)= & t^{0}\left(\mathbf{p}^{\prime}, \mathbf{p}\right) \delta_{\alpha \gamma} \delta_{\beta \delta} \\
& +t_{a}^{1}\left(\mathbf{p}^{\prime}, \mathbf{p}\right)\left(\sigma_{\alpha \gamma}^{a} \delta_{\beta \delta}+\delta_{\alpha \gamma} \sigma_{\beta \delta}^{a}\right) \\
& +t_{a b}^{2}\left(\mathbf{p}^{\prime}, \mathbf{p}\right) \sigma_{\alpha \gamma}^{a} \sigma_{\beta \delta}^{b},
\end{aligned}
$$

substituting in eq. (11), simplifying the Pauli matrices, and equating the coefficients of equal structures we arrive at the following system of equations:

$$
\begin{aligned}
t_{a b}\left(\mathbf{p}^{\prime}, \mathbf{p}\right)= & v_{a b}\left(\mathbf{p}^{\prime}, \mathbf{p}\right)-m^{2} \\
& \times \int \frac{\mathrm{d}^{3} \mathbf{k}}{(2 \pi)^{3}} W_{a b, x y}\left(\mathbf{p}^{\prime}, \mathbf{k}\right) G(\mathbf{k}) t_{x y}(\mathbf{k}, \mathbf{p}),
\end{aligned}
$$

where

$$
\begin{aligned}
& t_{a b}=\left(t^{0}, t_{a}^{1}, t_{a b}^{2}\right)^{\mathrm{T}}, \\
& v_{a b}=\left(v^{0}, v_{a}^{1}, v_{a b}^{2}\right)^{\mathrm{T}}, \\
& W_{a b, x y}= \\
& \left(\begin{array}{cc}
v^{0}, & 2 v_{x}^{1}, \\
v_{a}^{1}, & v^{0} \delta_{a x}+i \epsilon^{a m x} v_{m}^{1}+v_{a x}^{2}, \delta_{a x} v_{y}^{1}+i \epsilon^{a m x} v_{m y}^{2} \\
v_{a b}^{2}, & W_{32},
\end{array}\right), \\
& W_{32}=v_{a}^{1} \delta_{b x}+v_{b}^{1} \delta_{a x}+i \epsilon^{m x a} v_{m b}^{2}+i \epsilon^{m x b} v_{m a}^{2}, \\
& W_{33}=v^{0} \delta_{a x} \delta_{b y}-i \epsilon^{a x d} \delta_{b y} v_{d}^{1}-i \epsilon^{b y d} \delta_{a x} v_{d}^{1}-\epsilon^{m x a} \epsilon^{n y b} v_{m n}^{2}, \\
& G(\mathbf{k})=\frac{1}{\omega_{k}^{2}\left(E-2 \omega_{k}+i \epsilon\right)} .
\end{aligned}
$$

The amplitude of the deuteron interpolating field interacting with a pair of nucleon fields in the rest frame of the deuteron is given by

$$
D_{j}\left(\mathbf{p}^{\prime}\right)=\mathcal{P}_{j}+m^{2} \int \frac{\mathrm{d}^{3} \mathbf{k}}{(2 \pi)^{3}} \frac{T\left(\mathbf{p}^{\prime}, \mathbf{k}\right) \mathcal{P}_{j}}{\omega_{k}^{2}\left(E-2 \omega_{k}+i \epsilon\right)},
$$

where the LO NN scattering amplitude is obtained by solving eq. (11). It is convenient to parameterize the amplitude $D$ in terms of two structure functions $\Delta_{1}$ and $\Delta_{2}$ as

$$
D_{j}\left(\mathbf{p}^{\prime}\right)=\Delta_{1}\left(\mathbf{p}^{\prime 2}\right) \mathcal{P}_{j}+\mathbf{p}^{\prime}{ }_{a} \mathbf{p}_{b}^{\prime} \Delta_{2}\left(\mathbf{p}^{\prime 2}\right) \sigma^{a} \mathcal{P}_{j}\left(\sigma^{b}\right)^{T},
$$

where isospin indices and terms resulting from antisymmetrization are not shown explicitly. Notice that the structure functions $\Delta_{1}$ and $\Delta_{2}$ can be easily related to the $S$ - and $D$-state components of the deuteron wave 
function, see also ref. [25]. To derive equations for the structure functions $\Delta_{i}$ of the projected amplitude we parameterize the NN potential as

$$
\begin{aligned}
v^{0}\left(\mathbf{p}^{\prime}, \mathbf{p}\right)= & \nu_{1}\left(\mathbf{p}^{\prime}, \mathbf{p}\right), \\
v_{a}^{1}\left(\mathbf{p}^{\prime}, \mathbf{p}\right)= & i \epsilon^{a b c} \mathbf{p}^{b} \mathbf{p}^{\prime c} \nu_{3}\left(\mathbf{p}^{\prime}, \mathbf{p}\right), \\
v_{a b}^{2}\left(\mathbf{p}^{\prime}, \mathbf{p}\right)= & \delta_{a b} \nu_{2}\left(\mathbf{p}^{\prime}, \mathbf{p}\right)+\mathbf{p}^{\prime a} \mathbf{p}^{\prime b} \nu_{5}\left(\mathbf{p}^{\prime}, \mathbf{p}\right)+\mathbf{p}^{a} \mathbf{p}^{b} \nu_{6}\left(\mathbf{p}^{\prime}, \mathbf{p}\right) \\
& +\left(\mathbf{p}^{a} \mathbf{p}^{\prime b}+\mathbf{p}^{\prime a} \mathbf{p}^{b}\right) \nu_{4}\left(\mathbf{p}^{\prime}, \mathbf{p}\right),
\end{aligned}
$$

where the $\nu_{i}\left(\mathbf{p}^{\prime}, \mathbf{p}\right)$ are scalar functions of $\mathbf{p}^{\prime 2}, \mathbf{p}^{2}$ and $\mathbf{p}^{\prime} \cdot \mathbf{p}$. We then obtain the following system of integral equations:

$$
\begin{aligned}
\Delta_{1}\left(\mathbf{p}^{2}\right)= & +m^{2} \int \frac{\mathrm{d}^{3} \mathbf{k}}{(2 \pi)^{3}} G(\mathbf{k})\left\{\Delta _ { 1 } ( \mathbf { k } ^ { 2 } ) \left[\nu_{1}(\mathbf{p}, \mathbf{k})\right.\right. \\
& \left.+\nu_{2}(\mathbf{p}, \mathbf{k})+C_{1} \nu_{6}(\mathbf{p}, \mathbf{k})\right] \\
& +\Delta_{2}\left(\mathbf{k}^{2}\right)\left[C_{1}\left(\nu_{1}(\mathbf{p}, \mathbf{k})+\nu_{2}(\mathbf{p}, \mathbf{k})\right)\right. \\
& +2(\mathbf{p} \cdot \mathbf{k}) \nu_{3}(\mathbf{p}, \mathbf{k})+2 \mathbf{k}^{2}(\mathbf{p} \cdot \mathbf{k}) \nu_{4}(\mathbf{p}, \mathbf{k}) \\
& \left.\left.+\left[(\mathbf{p} \cdot \mathbf{k})^{2}-C_{1} \mathbf{p}^{2}\right] \nu_{5}(\mathbf{p}, \mathbf{k})+\left(\mathbf{k}^{2}\right)^{2} \nu_{6}(\mathbf{p}, \mathbf{k})\right]\right\} \\
\Delta_{2}\left(\mathbf{p}^{2}\right)= & m^{2} \int \frac{\mathrm{d}^{3} \mathbf{k}}{(2 \pi)^{3}} G(\mathbf{k})\left\{\Delta _ { 1 } ( \mathbf { k } ^ { 2 } ) \left[2 B \nu_{4}(\mathbf{p}, \mathbf{k})\right.\right. \\
& \left.+C_{2} \nu_{6}(\mathbf{p}, \mathbf{k})+\nu_{5}(\mathbf{p}, \mathbf{k})\right] \\
& +\Delta_{2}\left(\mathbf{k}^{2}\right)\left[C_{1} \nu_{5}(\mathbf{p}, \mathbf{k})-2 B \mathbf{k}^{2} \nu_{3}(\mathbf{p}, \mathbf{k})\right. \\
& \left.\left.+C_{2}\left(\nu_{1}(\mathbf{p}, \mathbf{k})+\nu_{2}(\mathbf{p}, \mathbf{k})+2(\mathbf{p} \cdot \mathbf{k}) \nu_{3}(\mathbf{p}, \mathbf{k})\right)\right]\right\}
\end{aligned}
$$

where we have defined

$$
\begin{aligned}
B & \equiv \frac{(\mathbf{p} \cdot \mathbf{k})}{\mathbf{p}^{2}}, \\
C_{1} & \equiv \frac{1}{2}\left[\mathbf{k}^{2}-\frac{(\mathbf{p} \cdot \mathbf{k})^{2}}{\mathbf{p}^{2}}\right], \\
C_{2} & \equiv \frac{3(\mathbf{p} \cdot \mathbf{k})^{2}-\mathbf{k}^{2} \mathbf{p}^{2}}{2\left(\mathbf{p}^{2}\right)^{2}} .
\end{aligned}
$$

As the $\Delta_{i}$ functions depend only on $\mathbf{k}^{2}$, the integration over angles can be carried out explicitly in eqs. (19) so that one is finally left with a system of two one-dimensional integral equations which can be solved numerically. The deuteron manifests itself as a pole at $P^{2}=E^{2}-\mathbf{0}^{2}=M_{d}^{2}$. Equations (19) are divergent and require regularization. Here, we employ cutoff regularization. However, since all ultraviolet divergences can be absorbed into a redefinition of the low-energy constant $C_{3} S_{1}=C_{S}+C_{T}$, we take the cutoff parameter $\Lambda$ to infinity after renormalization.

The three-point function is given by

$$
\begin{aligned}
& G_{i j}^{\mu}\left(P, P^{\prime}\right)=m^{3} \int \frac{\mathrm{d}^{3} \mathbf{k}}{(2 \pi)^{3}} \\
& \times \frac{D_{j, \alpha \beta}^{\dagger}\left(P^{\prime}, \mathbf{k}\right) \Gamma_{\alpha \beta, \alpha_{1} \beta_{1}}^{\mu}\left(P, P^{\prime}, \mathbf{k}\right) D_{i, \alpha_{1} \beta_{1}}(P, \mathbf{k})}{\omega_{k} \omega_{k-\frac{q}{2}} \omega_{k+\frac{q}{2}}\left(E-\omega_{k}-\omega_{k-\frac{q}{2}}\right)\left(E-\omega_{k}-\omega_{k+\frac{q}{2}}\right)},
\end{aligned}
$$

where $D_{j, \alpha \beta}^{\dagger}\left(P^{\prime}, \mathbf{k}\right)$ and $D_{i, \alpha_{1} \beta_{1}}\left(P, \mathbf{k}^{\prime}\right)$ denote the amplitudes of the deuteron interpolating field interacting with a pair of nucleons in a general frame. As appropriate at $\mathrm{LO}$, these quantities can be obtained from the amplitudes calculated in the rest frame of the deuteron, see eq. (16), by means of a Galilean transformation. We choose to work in the Breit frame, so that $D_{j}^{\dagger}\left(P^{\prime}, \mathbf{k}\right)=D_{j}^{\dagger}(\mathbf{k}+\mathbf{q} / 4)$ and $D_{i}(P, \mathbf{k})=D_{i}(\mathbf{k}-\mathbf{q} / 4)$.

Using eq. (17), the deuteron full propagator $G_{\mathcal{D}}$ can be written as

$$
G_{\mathcal{D}}(E, \mathbf{0})=m^{2} \int \frac{\mathrm{d}^{3} \mathbf{k}}{(2 \pi)^{3}} \frac{\Delta_{1}\left(\mathbf{k}^{2}\right)+\frac{\mathbf{k}^{2}}{3} \Delta_{3}\left(\mathbf{k}^{2}\right)}{\omega_{k}^{2}\left(E-2 \omega_{k}+i \epsilon\right)}+\text { N. P. }
$$

where "N.P." stands for contributions which do not contain the deuteron pole. We use eq. (22) to calculate the residue $Z$.

\section{Discussion and summary}

Using the formalism outlined above, we calculate the electromagnetic form factors of the deuteron at LO by solving the integral equations numerically. We employ exact isospin symmetry as appropriate at LO and use the following values for the low-energy constants entering the OPE potential:

$$
M_{\pi}=138 \mathrm{MeV}, \quad F_{\pi}=92.4 \mathrm{MeV}, \quad g_{A}=1.267 .
$$

The low-energy constant $C_{3} S_{1}$ is fixed to reproduce the experimental value of the deuteron binding energy of $B=2.22 \mathrm{MeV}$. The resulting description of neutronproton phase shifts and the quark mass dependence of the $S$-wave scattering lengths and the deuteron binding energy can be found in refs. [6] and [30], respectively ${ }^{2}$. In particular, the ${ }^{3} S_{1}$ and ${ }^{3} D_{1}$ phase shifts and the mixing angle $\epsilon_{1}$ turn out to be reasonably well described (at least) up to laboratory energies of the order of $E_{\text {lab }} \sim 250 \mathrm{MeV}$. The corresponding parameter-free and cutoff-independent predictions for the electromagnetic form factors of the deuteron are plotted in fig. 2. In all cases the obtained results agree reasonably well with experimental data in the whole plotted range of the momentum transfer. For low values of $q$ all three form factors are accurately predicted at LO with the deviations increasing at high momentum transfers and reaching about $\sim 20 \%$ at $q=200 \mathrm{MeV}$. Similarly to observations made in earlier studies based on the non-relativistic approach $[11,12,24]$, the deviations from the experimental data can be largely traced back to the slow convergence of the chiral expansion of the nucleon form factors [31,32]. Indeed, substituting the phenomenological parametrization of the nucleon form factors from ref. [33], our predictions for $F_{C}\left(q^{2}\right), F_{M}\left(q^{2}\right)$ and $F_{Q}\left(q^{2}\right)$ are in an excellent agreement with the data even at large values of $q$. For the deuteron magnetic and quadrupole mo-

\footnotetext{
${ }^{2}$ Note that in ref. [6], the low-energy constant was adjusted to the empirical phase shifts rather then to the deuteron binding energy. This leads, however, only to a minor difference in the produced nucleon-nucleon scattering amplitude.
} 

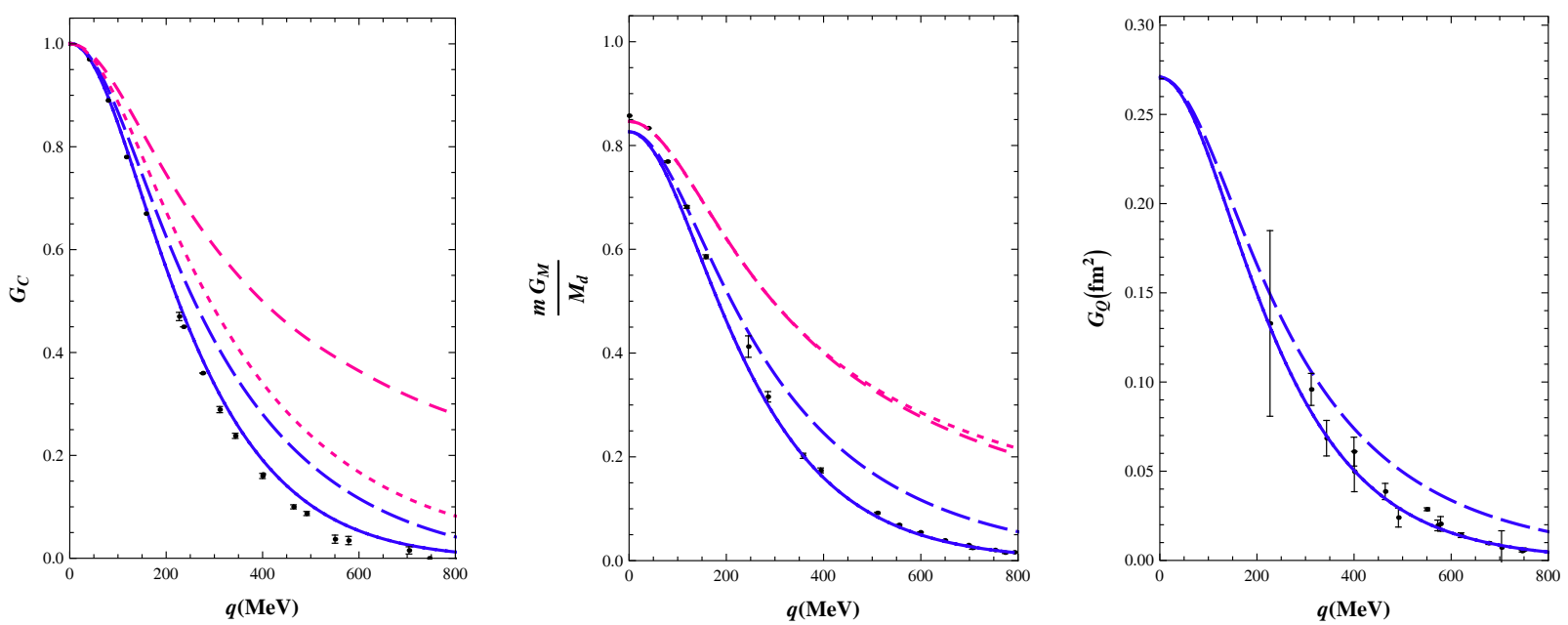

Fig. 2. LO EFT predictions for the Coulomb (left panel), magnetic (middle panel) and quadrupole (right panel) form factors of the deuteron as a function of the momentum transfer $q$ in comparison with experimental data from refs. [34,35]. Solid and long-dashed lines (short- and medium-dashed lines) show the predictions in the chiral (pionless) EFT with and without using phenomenological form factors of the nucleon, respectively.
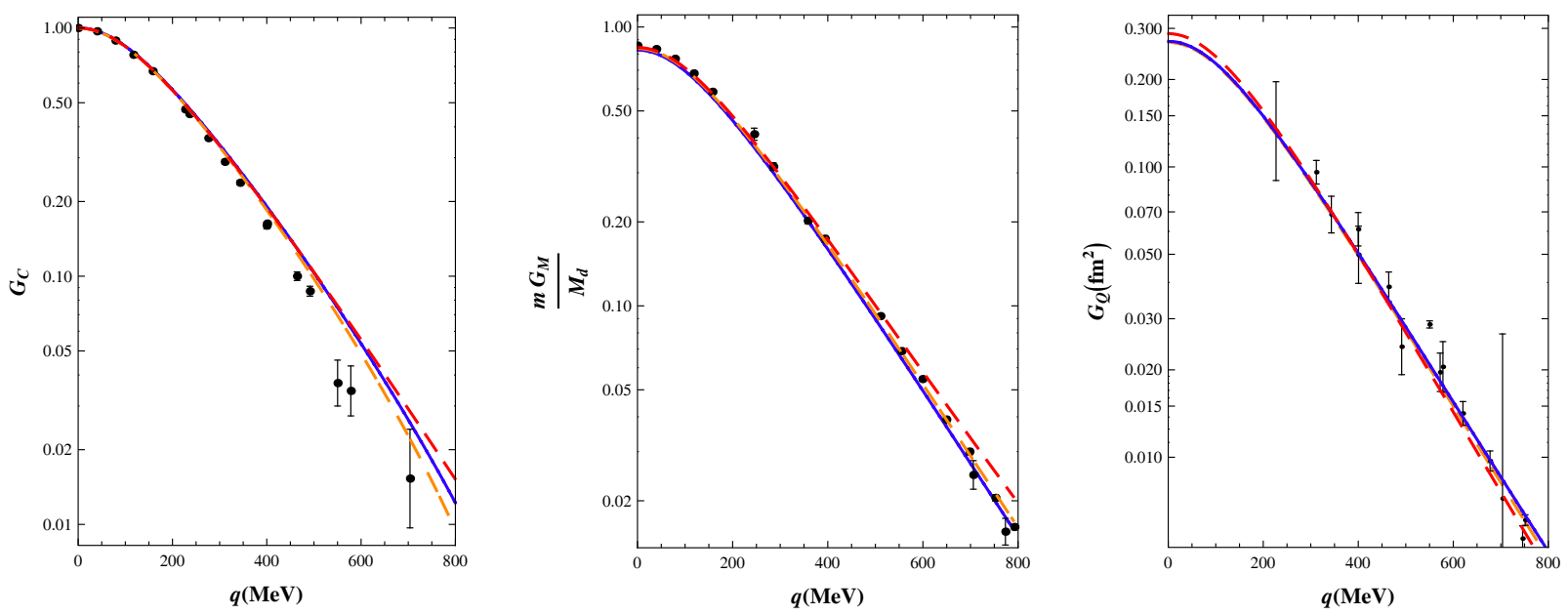

Fig. 3. LO EFT predictions for the Coulomb (left panel), magnetic (middle panel) and quadrupole (right panel) form factors of the deuteron as a function of the momentum transfer $q$ in comparison with experimental data from refs. [34,35]. Solid(blue), dashed(red) and dashed-dotted(orange) lines show the predictions of the current work (solid line in Fig. 2) and of the nonrelativistic approach with cutoff values 800 and $20000 \mathrm{MeV}$, respectively.

ments we obtain the values of $\mu_{M}^{\mathrm{LO}}=0.826(e /(2 m))$ and $\mu_{Q}^{\mathrm{LO}}=0.271 \mathrm{fm}^{2}$ in good agreement with the experimental numbers of $\mu_{M}=0.85741(e /(2 m))$ and $\mu_{Q}=0.2859 \mathrm{fm}^{2}$. The observed deviations of the order of $\sim 3.5 \%$ for the magnetic moment and $\sim 5 \%$ for the quadrupole moment are consistent with the expected size of higher-order corrections due to the two-nucleon currents $[13,23]$.

It is also instructive to compare the results in the EFT with and without explicit pions, see fig. 2. The pionfull approach yields a clearly superior description of the Coulomb and magnetic form factors. This is consistent with the observation that the one-pion-exchange potential plays a very important role in the ${ }^{3} S_{1}{ }^{3} D_{1}$ channel as witnessed, e.g., by the low-energy theorems, see ref. [6]. Notice that the quadrupole form factor vanishes at LO in the pionless approach since the deuteron in this case does not have a D-state component. Finally, we emphasize that our pion- less results agree well with the ones obtained within the non-relativistic framework in ref. [8].

We also performed deuteron form factor calculations in non-relativistic EFT applying cutoff regularization. The results are plotted together with the results of the current work and the experimental data in fig. 3. A comparison between our results and the ones obtained within the nonrelativistic framework with a finite cutoff $(\Lambda \sim 800 \mathrm{MeV})$ shows that relativistic effects and finite-cutoff artifacts are numerically small for the form factors at low values of momentum transfer. Such agreement is to be expected given the highly non-relativistic nature of the deuteron bound state. The predictions for the form factors in the non-relativistic framework of refs. [36,37] employing an infinitely large cutoff, are also close to our results ${ }^{3}$.

${ }^{3}$ For our interpretation of such an approach, see refs. [38,6]. 
To summarize, in the present work we calculated the electromagnetic form factors of the deuteron at LO in an EFT using the renormalizable approach of ref. [6]. Following ref. [8], we introduced an interpolating field for the deuteron and calculated the form factors by applying the LSZ reduction formalism to the three-point correlation function. We worked out a set of integral equations (without making use of partial wave decomposition), which are renormalizable at $\mathrm{LO}$, i.e. all ultraviolet divergences are absorbable into a redefinition of the parameters of the LO potential. Our parameter-free and cutoff-independent predictions for the electromagnetic form factors of the deuteron at LO are in very good agreement with the experimental data.

MRS thanks S. Pastore for useful discussions on electromagnetic current operators. This work was supported in part by Georgian Shota Rustaveli National Science Foundation (grant 11/31), DFG (SFB/TR 16, "Subnuclear Structure of Matter"), by the European Community-Research Infrastructure Integrating Activity "Study of Strongly Interacting Matter" (acronym HadronPhysics3, Grant Agreement n. 283286) under the Seventh Framework Programme of EU, ERC project 259218 NUCLEAREFT, and the US Department of Energy under grant no. DE-SC0010300.

Open Access This is an open access article distributed under the terms of the Creative Commons Attribution License (http://creativecommons.org/licenses/by/4.0), which permits unrestricted use, distribution, and reproduction in any medium, provided the original work is properly cited.

\section{References}

1. S. Weinberg, Phys. Lett. B 251, 288 (1990).

2. S. Weinberg, Nucl. Phys. B363, 3 (1991).

3. C. Ordonez, U. van Kolck, Phys. Lett. B 291, 459 (1992).

4. E. Epelbaum, H.-W. Hammer, U.-G. Meißner, Rev. Mod. Phys. 81, 1773 (2009).

5. R. Machleidt, D.R. Entem, Phys. Rep. 503, 1 (2011).

6. E. Epelbaum, J. Gegelia, Phys. Lett. B 716, 338 (2012).

7. V.G. Kadyshevsky, Nucl. Phys. B 6, 125 (1968).

8. D.B. Kaplan, M.J. Savage, M.B. Wise, Phys. Rev. C 59, 617 (1999).

9. D.R. Phillips, T.D. Cohen, Nucl. Phys. A 668, 45 (2000).

10. M. Walzl, U.-G. Meißner, Phys. Lett. B 513, 37 (2001).
11. D.R. Phillips, Phys. Lett. B 567, 12 (2003).

12. D.R. Phillips, J. Phys. G 34, 365 (2007).

13. S. Kölling, E. Epelbaum, D.R. Phillips, Phys. Rev. C 86 , 047001 (2012).

14. C. Ordonez, L. Ray, U. van Kolck, Phys. Rev. C 53, 2086 (1996).

15. T.-S. Park, K. Kubodera, D.-P. Min, M. Rho, Phys. Rev. C 58, 637 (1998).

16. T.-S. Park, D.-P. Min, M. Rho, Nucl. Phys. A 596, 515 (1996).

17. S. Pastore, R. Schiavilla, J.L. Goity, Phys. Rev. C $\mathbf{7 8}$, 064002 (2008).

18. S. Pastore, L. Girlanda, R. Schiavilla, M. Viviani, R.B. Wiringa, Phys. Rev. C 80, 034004 (2009).

19. S. Pastore, L. Girlanda, R. Schiavilla, M. Viviani, Phys. Rev. C 84, 024001 (2011).

20. S. Kölling, E. Epelbaum, H. Krebs, U.-G. Meißner, Phys. Rev. C 80, 045502 (2009).

21. S. Kölling, E. Epelbaum, H. Krebs, U.-G. Meißner, Phys. Rev. C 84, 054008 (2011).

22. D. Rozpedzik, J. Golak, S. Kölling, E. Epelbaum, R. Skibinski, H. Witala, H. Krebs, Phys. Rev. C 83, 064004 (2011).

23. M. Piarulli, L. Girlanda, L.E. Marcucci, S. Pastore, R. Schiavilla, M. Viviani, Phys. Rev. C 87, 014006 (2013).

24. M.P. Valderrama, A. Nogga, E. Ruiz Arriola, D.R. Phillips, Eur. Phys. J. A 36, 315 (2008).

25. I. Fachruddin, C. Elster, W. Glöckle, Phys. Rev. C 63, 054003 (2001).

26. M.J. Zuilhof, J.A. Tjon, Phys. Rev. C 22, 2369 (1980).

27. P.J. Mohr, B.N. Taylor, D.B. Newell, Rev. Mod. Phys. 84, 1527 (2012).

28. D.M. Bishop, L.M. Cheung, Phys. Rev. A 20, 381 (1979).

29. T.E.O. Ericson, M. Rosa-Clot, Nucl. Phys. A 405, 497 (1983).

30. E. Epelbaum, J. Gegelia, PoS CD 12, 090 (2013).

31. B. Kubis, U.-G. Meißner, Nucl. Phys. A 679, 698 (2001).

32. T. Bauer, J.C. Bernauer, S. Scherer, Phys. Rev. C 86, 065206 (2012).

33. M.A. Belushkin, H.-W. Hammer, U.-G. Meißner, Phys. Rev. C 75, 035202 (2007).

34. JLAB t20 Collaboration (D. Abbott et al.), Eur. Phys. J. A 7, 421 (2000).

35. D.M. Nikolenko et al., Phys. Rev. Lett. 90, 072501 (2003).

36. S.R. Beane, P.F. Bedaque, M.J. Savage, U. van Kolck, Nucl. Phys. A 700, 377 (2002) nucl-th/0104030.

37. A. Nogga, R.G.E. Timmermans, U. van Kolck, Phys. Rev. C 72, 054006 (2005).

38. E. Epelbaum, J. Gegelia, Eur. Phys. J. A 41, 341 (2009). 